prolongation and increased incidence of pro-arrhythmic events in isolated cardiomyocytes which was prevented by pharmacological inhibition of the late sodium current. Our current data suggests a direct effect of the mutation on cardiac function rather than the observed phenotypes resulting from an accumulation of BCAAs. Thus we have identified a novel model of sudden cardiac death resulting from abnormal BCAA metabolism.

\section{T4 ERK5 DEGRADATION: A TURNING POINT FROM COMPENSATED METABOLIC CARDIOMYOPATHY TO HEART FAILURE}

A Ruiz-Velasco, W Liu, X Wang. Faculty of Biology, Medicine, and Health, The University of Manchester, Oxford Rd, Manchester, UK

\subsection{6/heartjnl-2018-BSCR.4}

Rationale The accumulated prevalence of obesity, diabetes, and metabolic syndrome is more than $25 \%$ of the world's population. These are all conditions that have been repeatedly related to a higher risk of heart failure, and effective treatment has not been found. It remains essential to continue deciphering the underlying molecular mechanism to discover novel treatment strategies.

Methodology Initial screening was performed on myocardium samples from ob/ob mice, $\mathrm{db} / \mathrm{db}$ mice, rhesus monkey with spontaneous metabolic syndrome, and mice fed for 25 weeks with high-fat diet (HFD). In subsequent studies, extracellular signal-regulated protein kinase 5 (ERK5) cardiomyocyte-specific knockout mice (ERK5-cko) were evaluated up until 16 weeks of HFD feeding. In vitro experiments were performed on rat ventricular myocytes treatd with saturated fatty acids.

Results The screening of obese and diabetic models showed that ERK5 was selectively lost in the myocardium. ERK5-cko presented cardiac dysfunction after only 16 weeks of HFD. Further studies showed the loss of contractility was accompanied by augmented oxidative stress, increased lipid accumulation, and severe mitochondrial dysfunction. Mechanistic studies revealed ERK5 to act upstream of the mitochondrial regulator peroxisome proliferator-activated receptor $\gamma$ co-activator-1 $\alpha$ (PGC-1 $\alpha)$. Moreover, it was observed that ERK5 degradation after saturated fatty acid treatment was mediated by calpain-1, while the inhibition of this degradation could prevent the mitochondrial dysfunction.

Conclusion The calpain-mediated degradation of ERK5 blunts the compensatory response that would usually maintain mitochondrial integrity when facing metabolic stress.

\section{\begin{tabular}{|l|l}
\hline T5 & VERY LOW CALORIE DIET IN OBESITY IMPROVES
\end{tabular} METABOLIC RISK FACTORS AT THE INITIAL COST OF VENTRICULAR FUNCTION AND STEATOSIS}

JJ Rayner, MA Peterzan, S Neubauer, OJ Rider. OCMR, Division of Cardiovascular Medicine, University of Oxford

\subsection{6/heartjnl-2018-BSCR.5}

Background Very Low Calorie Diets (VLCD) in obesity are an effective weight loss intervention that rapidly reduce liver fat and improve peripheral insulin resistance. We hypothesised that improved peripheral glycaemic control would be accompanied by initial impairment of cardiac function as hepatic fat stores are mobilised and taken up by the myocardium. Methods 14 obese volunteers (4 male, $49 \pm 15$ years, BMI $36.2 \pm 5.9 \mathrm{~kg} \mathrm{~m}-2$ ) underwent body composition analysis and MR scanning for abdominal visceral and liver fat, LV structure and function, $1 \mathrm{H}-\mathrm{MRS}$ to measure myocardial triglyceride content (MTGC), and echocardiography for diastolic function (E/E'), before and one week into a VLCD (800 kcal/day).

Results 7 days of VLCD led to significant reductions in total body fat, visceral and hepatic fat, and insulin resistance. However, MTGC rose from $1.74 \pm 0.99 \%$ to $3.02 \pm 1.70 \%$ $(\mathrm{p}=0.030)$, and there was a reduction in both systolic function (LVEF $67 \pm 3 \%$ to $62 \pm 5 \%, \mathrm{p}=0.014$; peak radial strain 51 $\pm 8 \%$ to $42 \pm 9 \%, \mathrm{p}=0.005)$ and diastolic function (e/e' 8.5 \pm 1.6 to $10.3 \pm 3.5, \mathrm{p}=0.034)$. The change in MTGC at one week correlated with change in diastolic function $(r=0.729$, $\mathrm{p}=0.017)$. However at 8 weeks $(n=6)$, changes in MGTC as well as cardiac function had returned to normal.

Conclusions We demonstrate for the first time in healthy obese individuals that a 7 day period of severe caloric restriction results in accumulation of myocardial fat which is associated with impairment of both systolic and diastolic LV function at this timepoint, despite a significant improvement in hepatic fat and whole body insulin sensitivity.

\section{P1 CAUSAL LINK BETWEEN INTRACELLULAR SODIUM OVERLOAD AND METABOLIC REMODELLING IN THE HEART: UNCOUPLING ATP SUPPLY AND DEMAND?}

${ }^{1} \mathrm{D}$ Aksentijević, ${ }^{2} \mathrm{~A}$ Karlstädt, ${ }^{1} \mathrm{M}$ Basalay, ${ }^{1} \mathrm{BA}$ O'Brien, ${ }^{3} \mathrm{~A}$ Thakker, ${ }^{3} \mathrm{D}$ Tennant, ${ }^{4} \mathrm{~W}$ Fuller, ${ }^{2} \mathrm{H}$ Taegtmayer, ${ }^{5} \mathrm{TR}$ Eykyn, ${ }^{1} \mathrm{MJ}$ Shattock. 'British Heart Foundation Centre of Research Excellence, King's College London, The Rayne Institute, St Thomas' Hospital, London, UK; ${ }^{2}$ Department of Internal Medicine, Division of Cardiology, The University of Texas Health Science Centre at Houston, McGovern Medical School, Houston, Texas, USA; ${ }^{3}$ Institute of Metabolism and Systems Research, College of Medical and Dental Sciences University of Birmingham, Edgbaston, Birmingham, UK; ${ }^{4}$ Division of Cardiovascular and Diabetes Medicine, Medical Research Institute, College of Medicine, Dentistry and Nursing, University of Dundee, Ninewells Hospital and Medical School, Dundee, UK; ${ }^{5}$ Department of Imaging Chemistry and Biology, Division of Imaging Sciences and Biomedical Engineering, King's College London, St Thomas' Hospital, London, UK

\subsection{6/heartjnl-2018-BSCR.6}

Rationale Intracellular $\mathrm{Na}$ elevation is a hallmark of the ischaemic and failing heart - pathologies in which both acute and chronic metabolic remodelling occur.

Objective To assess whether acute $(75 \mu \mathrm{M}$ ouabain $100 \mathrm{nM}$ blebbistatin) and chronic myocardial Nai load (PLM3SA mouse) are causally linked to metabolic remodelling and if the failing heart shares a common Na-mediated metabolic 'fingerprint'.

Methods 23Na, 31P and 13C NMRS were performed in normal and hypertrophied (pressure overload) Langendorff perfused mouse hearts followed by 1 hour NMRS metabolomic profiling, mass spec and in silico modelling.

Results Na overload (acute, chronic (PLM3SA), and hypertrophy 2, 1.3 and 1.4-fold respectively) resulted in common metabolic perturbations: substrate switch (palmitate 35\% reduction, glucose 58\% increase), flux (TCA cycle, OXPHOS, glycolysis) and metabolomic profile (TCA cycle, glycolysis, anaplerosis) without energetic impairment (PCr/ATP 1.5 \pm 0.1 vs control $1.3 \pm 0.1$ ). Inhibition of mitochondrial $\mathrm{Na} / \mathrm{Ca}$ exchanger by CGP37157 during both acute and chronic $\mathrm{Na}$ load ameliorated the metabolic changes. 